\title{
Dyspareunia in Female Fibromyalgia patients
}

\author{
Original \\ Article
Mohamed Ahmed Hefny ${ }^{1}$, Mai Mohamed Abdelnaby ${ }^{1}$, Nermeen Hassan Abdel Moneim $^{1}$, Mohamed Azab ${ }^{2}$ and Hanan Mohamed Ghoneim ${ }^{3}$ \\ ${ }^{1}$ Department of Physical Medicine Rheumatology and Rehabilitation, ${ }^{2}$ Department of \\ Dermatology, Venereology and Department of Andrology, ${ }^{3}$ Gynecology \& Obstetrics, Faculty \\ of Medicine, Suez Canal University Hospital
}

\begin{abstract}
Objective: To evaluate tender points number, pressure pain threshold and incidence of fibromyalgia in women with dyspareunia

Patients and Methods: Married women dyspareunia were compared to 35 healthy controls. Females were asked if they encountered sexual intercourse throughout the preceding month and dyspareunia was rated from zero to 3 by using Marinoff Dyspareunia Scale (MDS). Dolorimeter (pressure algometer) was employed to assess pressure pain threshold. Diagnosis of Fibromyalgia was based on the 1990 American College of Rheumatology (ACR) criteria. Depression status of was assessed using the Beck Depression Inventory (BDI).

Results: No significant statistical difference was found among both patients and control groups in relation to age, body mass index (BMI). Myalgic score and total tender point score was significantly lower in the dyspareunia group. Tender points score among dyspareunia patients was significantly higher. The

mean score of Beck Depression Inventory (BDI) was 18.1 \pm 6.7 among dyspareunia group diagnosed with fibromyalgia compared to $11.3 \pm 4.1$ in the dyspareunia group without fibromyalgia

Conclusion: fibromyalgia is a contributing factor to dyspareunia
\end{abstract}

Key Words: Dyspareunia, fibromyalgia

Received: 11 July 2019, Accepted: 14 August 2019

Corresponding Author: Hanan Mohamed Ghoneim, MD, Department of Gynecology and Obstetrics, Faculty of Medicine, Suez Canal university ,Ismailia, Egypt, Tel.: +201025561955, E-mail: hananghoneim@gmail.com

ISSN: 2090-7265, August 2019, Vol. 9, No. 3

\section{INTRODUCTION}

Fibromyalgia syndrome is musculoskeletal conditionpatented by diffuse musculoskeletal pain, morning stiffness, easy fatigability and the presence of multiple bodily tender points. It occurs in about $1.7 \%$ in the general population. Women are affected mainly with Fibromyalgia with a female: male ratio of $13.7: 1^{[1]}$. Fibromyalgia (FM) syndrome is one of the most common musculoskeletal disorders with unknown cause involving adults especially women aged $20-55^{[2]}$. The condition is associated with a marked decrease in functional capacity and profoundly interferes with activities of daily living through affecting the individual's social performance ${ }^{[3]}$. Much other comorbidity can be associated with fibromyalgia, such as chronic fatigue syndrome, irritable bowel syndrome, dysmenorrhea, migraine, restless leg syndrome and affective disorders, all of them share a common central pathophysiology ${ }^{[4]}$.

Dyspareunia is defined as recurring or persistent pain due to sexual activity causing marked distress or interpersonal conflict ${ }^{[5]}$. It may be classified as entry or deep. Entry dyspareunia is a pain during initial or attempted penetration of the vaginal introitus, whereas deep dyspareunia occurs with deep vaginal penetration.
Dyspareunia is also classified as primary (i.e., occurring with sexual debut and thereafter) or secondary (i.e., beginning after previous sexual activity that was not painful $)^{[6]}$. Definingif dyspareunia is entry or deep can point to detailed causes, although the primary vs. secondary classification is less likely to narrow the differential diagnosis. Dyspareunia prevalence is approximately 10$28 \%$ in U.S. Women with the leading causes varying by age group ${ }^{[7]}$. The pain associated with dyspareunia results in peripheral sensitization of neuropathic nature as the process induces central sensitization ${ }^{[8]}$. There is a strong relationship between dyspareunia and fibromyalgia as dyspareunia is accompanied by a condition called vestibulodynia with increased irritated areas in the vulva ${ }^{[8]}$. this vulvodynia causes hyperactivity in the corresponding central sites stimulated by pressure ${ }^{[9]}$.

Our study objectiveswere to evaluate tender points and pressure pain threshold and the incidence of fibromyalgia in femalesubjects with dyspareunia and comparing them with healthy individuals.

\section{PATIENTS AND METHODS}

This study was carried out at the Obstetrics and Gynecology outpatient clinic in association with Physical 
Medicine, Rheumatology and Rehabilitation and Dermatology, Venereology and Andrology departmentsat Suez Canal University Hospital. Study population was composed of married females attending the gynecology clinic and they are asked if they had had sexual intercourse during the preceding 4 weeks, and asked if they have dyspareunia, we divided those participants into 2 groups. Patients group which include 51 individuals with dyspareunia. And the other group contained 35 females with no dyspareunia. Women aged between 20 and 45 years old were included in this study. Pregnant females, patients with a diagnosed organic cause of dyspareunia (e.g. Pelvic surgery, endometriosis or pelvic inflammatory disease) were excluded from this study. A detailed medical and drug histories were obtained; a full clinical examination was performed. All included individuals underwent assessment for fibromyalgia according to 1990 diagnostic criteria of the American college of rheumatology ${ }^{[10]}$. Dyspareunia was recorded and graded on a $0-3$ score according to the Marinoff Dyspareunia Scale ${ }^{[1]}$.

We used the visual analogue (VAS) scale to assess the severity of dyspareunia. The VAS consists of a straight line with the endpoints defining extreme limits such as no pain at all and 'pain as bad as it could be. The patient is asked to mark his pain level on the line between the two endpoints. The distance between 'no pain at all' and the mark then defines the subject's pain ${ }^{[12]}$. Pressure pain threshold (PPT) is the minimal pressure quantity where pressure first changes to pain. Pressure was induced using a pressure algometer (PainTest ${ }^{\mathrm{TM}}$ FPN 100 Algometer (Wagner Instruments, Greenwich, USA)) with a flat circular metal probe dressed in a rubber cover with a surface area of $1 \mathrm{~cm}$ applied to median, ulnar, radial, and c5-6 zygapophyseal joint. Algometer was mounted vertically and the pressure was increased. Patients were asked to notify the investigator when they start to feel pain (pain threshold). For each measurement the algometer was calibrated to enable force to be applied at a controlled and steady rate, the mean of three trials (intra-examiner reliability) were calculated and used for main analysis. A 30 secresting period was allowed between each measure. We used pressure algometer in the 18 bilateral tender points (insertion of the suboccipital muscle, upper midpoint of the trapezius muscle, origin of the supraspinatus muscle, lower part of the sternocleidomastoid muscle, second costochondral junction, $2 \mathrm{~cm}$ distal to the lateral epicondyle, upper lateral part of the gluteal region, surface of the greater trochanter and the medial fat pad of the knee) described in the 1990 American College of Rheumatology criteria for fibromyalgia ${ }^{[12]}$. Painful pressures sum in kilograms (tender points) was documented as the myalgic score, and the sum of painful pressures in kilograms in the 4 control points was recorded as the control point score. The mean tender point pressure pain threshold was calculated by dividing the sum of minimal pressure that induced pain during dolorimetric measurement in the tender points to the number of painful points.

In the present study the Beck Depression Inventory (BDI) was used to assess depression. It is a subjective depression scale with 21 items. The summation of scores ranges from 0 to 63 ; big scores indicate severe depression. The score analysis is classified as follows: 0 to $13=$ no depression, 14 to $24=$ mild depression and $>25=$ severe depression ${ }^{[13]}$.

\section{RESULTS}

A total of 51 femalepatients with dyspareunia and 35 controls were enrolled in the study. No statistically significant difference was found between the patient, and the control groups as for ages,body mass index. The socio demographic data summarized in table 1

The mean duration of dyspareunia was $18.3 \pm 4.1$ months. According to Marinoff Dyspareunia Scale 12 patients had grade 1,17patients had grade 2 and 22patientshade grade 3 dyspareunia. Total myalgic score, total control score and tender point pain score threshold was significantly lower in the dyspareunia group compared with that of the control group. The tender point count was significantly higher in the dyspareunia group. The Beck Depression Inventory score was $14.7 \pm 8.4$ in the dyspareunia group and $11.2 \pm 7.1$ in the control group. No statistical difference was found significant (Table 1).Nine patients $(17.6 \%$ ) with dyspareunia were diagnosed fibromyalgia, whereas no patients in the control group were diagnosed fibromyalgia. The diagnosis of fibromyalgia was established according to the ACR Criteria $2010^{[10]}$. There was no significant difference between the two groups in terms of the presence of fibromyalgia $(\mathrm{P}=0.09)$.

The mean age of the group with dyspareunia and fibromyalgia was $38.9 \pm 2.1$ years, compared with $38.7 \pm 2.3$ years in the group with dyspareunia without fibromyalgia. The difference between the groups in terms of age was not statistically significant $(\mathrm{P}=0.14)$. The mean Beck Depression Inventory score was 18.1 \pm 6.7 in the dyspareunia group with fibromyalgia compared with $11.3 \pm 4.1$ in the dyspareunia group without fibromyalgia. The difference was statistically significant $(\mathrm{P}=0.03)$. There was no significant difference between the groups with regard to dyspareunia grade $(\mathrm{P}=0.2)$. The mean dyspareunia VAS score was $6.02 \pm 2.1$ in patients with fibromyalgia and $4.12 \pm 1.1$ in patients without fibromyalgia. There was a 
significant difference in dyspareunia VAS scores between the two groups $(\mathrm{P}=0.04)$ (Table 2$)$.

Table 1: Socio- Demographic characteristics and Clinical features of the studied and control group.

\begin{tabular}{|c|c|c|c|}
\hline Character & $\begin{array}{c}\text { Dyspareunia } \\
(\mathrm{n}=51)\end{array}$ & $\begin{array}{l}\text { Control group } \\
\quad(\mathrm{n}=35)\end{array}$ & $\mathrm{P}$ \\
\hline Age(years) & & & 0.5 \\
\hline Mean $\pm \mathrm{SD}$ (range) & $34.2 \pm 9.9[19-56]$ & $40 \pm 15.32$ & \\
\hline \multicolumn{4}{|l|}{ Education (n \%) } \\
\hline Read and write & $8(15.7)$ & $7(20)$ & \\
\hline Elementary & $11(21.6)$ & $3(8 \%)$ & \\
\hline Preparatory & $18(35.3)$ & $11(32 \%)$ & \\
\hline Secondary & $12(23.5)$ & $14(40 \%)$ & 0.03 \\
\hline Postgraduate & $2(3.9)$ & $3(6 \%)$ & \\
\hline \multicolumn{4}{|l|}{ Occupation (n \%) } \\
\hline Employed & $44(10 \%)$ & $7(20 \%)$ & \\
\hline Unemployed & $7(78.6 \%)$ & $27(77 \%)$ & \\
\hline Retired & 0 & $1(3 \%)$ & \\
\hline \multicolumn{4}{|l|}{ Marital Status (n \%) } \\
\hline Married & $41(81 \%)$ & $33(94 \%)$ & \\
\hline Divorced/separated & $10(29 \%)$ & $2(6 \%)$ & \\
\hline Smoking (n \%) & & & 0.1 \\
\hline Smoker & $0(0 \%)$ & $0(0 \%)$ & \\
\hline Non-Smoker & $51(100 \%)$ & $(100 \%)$ & \\
\hline BMI, $\mathrm{kg} / \mathrm{m}^{2}$ & $27.5 \pm 5.2$ & $28.3 \pm 4.01$ & 0.6 \\
\hline BDI score & $14.7 \pm 8.4$ & $11.2 \pm 7.1$ & 0.1 \\
\hline $\begin{array}{l}\text { Total myalgic score, } \\
\mathrm{g} / \mathrm{cm}^{2}\end{array}$ & $133.2 \pm 18.6$ & $164.2 \pm 9.2$ & $0.02 *$ \\
\hline $\begin{array}{l}\text { Total control score, } \\
\mathrm{g} / \mathrm{cm}^{2}\end{array}$ & $22.4 \pm 3.7$ & $27.5 \pm 3.4$ & $0.03 *$ \\
\hline Tender point count & $4.7 \pm 3.5$ & $1.5 \pm 1.1$ & $0.01 *$ \\
\hline $\begin{array}{l}\text { Tender point mean } \\
\text { pain threshold }\end{array}$ & $2.8 \pm 0.54$ & $3.9 \pm 1.0$ & $0.03 *$ \\
\hline
\end{tabular}

Table 2: Grade of dyspareunia and pain scores in fibromyalgia group in patients with dyspareunia

\begin{tabular}{|c|c|c|c|}
\hline \multirow[t]{2}{*}{ Variable } & \multicolumn{2}{|c|}{ Dyspareunia } & \multirow[t]{2}{*}{$\mathrm{P}$} \\
\hline & $\begin{array}{c}+ \text { Fibromyalgia } \\
(\mathrm{n}=9)\end{array}$ & $\begin{array}{c}\text { - Fibromyalgia } \\
(\mathrm{n}=42)\end{array}$ & \\
\hline Age, years, mean $\pm \mathrm{SD}$ & $38.9 \pm 2.1$ & $38.7 \pm 2.3$ & 0.1 \\
\hline $\begin{array}{l}\text { Marinoff Dyspareunia } \\
\text { Scale }\end{array}$ & & & \\
\hline Grade 1 & $1(11.1)$ & $11(26.1)$ & 0.2 \\
\hline Grade 2 & $4(44.4)$ & $13(30.9)$ & \\
\hline Grade 3 & $4(44.4)$ & $18(42.8)$ & \\
\hline $\begin{array}{l}\text { Visual analogue scale } \\
\text { score, mean } \pm \text { SD }\end{array}$ & $6.02 \pm 2.1$ & $4.12 \pm 1.1$ & $0.04 *$ \\
\hline
\end{tabular}

Data presented as $\mathrm{n}(\%)$ unless otherwise indicated.*Statistically significant $(\mathrm{P}<0.05)$. + With; - Without

\section{DISCUSSION}

Multiple epidemiologic studies have demonstrated an increased prevalence for women in several chronic pain disorders. Clinical and experimental investigations have consistently demonstrated sex-specific differences in pain sensitivity and pain threshold ${ }^{[14]}$. Recent researches have shown sex-specific differences in pain threshold andsensitivity. Although the specific pathogenic mechanism responsible for that difference has not yet been identified, the possibilityof the effect of sex hormone on the nociceptive process has attracted attention ${ }^{[14]}$.

There are structural and functional abnormalities in the peripheral sensory nerves, especially in the vulvar and vestibular tissues ${ }^{[15]}$. There is a theory shown that the peripheral sensitization in patients of dyspareunia is considered to induce a central sensitization with time ${ }^{[8]}$. Thus, pain sensitivity in those patients may generalize to non-genital parts ${ }^{[16]}$. Provoked vestibulodynia characterized by painful and chronic inflammation of vestibular structures is the most common causes ofdyspareunia ${ }^{[17]}$.

On the current study, pressure pain threshold was lower in the patients with dyspareuniaat boththe tender and control points, and fibromyalgia was identified in the dyspareunia group than the control patients.

The Total myalgic score and Beck Depression Inventory score was significantly higher in patients with dyspareunia diagnosed with fibromyalgia compared with patients with dyspareunia without fibromyalgia.

Granot et al, evaluated the heat pain administered to the forearms to assess the pain thresholds, and also to assess the level of pain associated with suprathreshold stimuli. lower thresholds for pain and discomfort were found among vulvar vestibulitis patients, as well as a suprathreshold for pain that was 13 times more than that in the control group $^{[18]}$.

In a study conducted by Pukall et al, evaluating generalized pain sensitivity in 16 vulvar vestibulitis patients with genital pain and 16 controls, more tender points were found inpatients with vulvar vestibulitis compared to that in thecontrol group. Moreover, those patients had a considerable higher level of pain. And this indicate that development of vulvar vestibulitis may had other mechanisms of actions that are not limited to the genitals, and may have a more centralized nature ${ }^{[19]}$.

Beck Depression Inventory score was significantly higher in the dyspareunia patients diagnosed with fibromyalgia that the group without fibromyalgia. Literature reviews had reported a positive correlation between the painful conditions such as fibromyalgia and dyspareunia and the severity of depression and pain ${ }^{[20]}$. Also, the occurrence of two painful conditions - dyspareunia and fibromyalgia may have led to higher depression scores.

In the current study, the pressure pain threshold at tender points was lower in patients with dyspareunia than that in the control group. This finding supports the theory that pain sensitivity may not be limited to the genital area; central mechanisms may be involved in its pathogenesisand alsothere are interactions in pain modulation. 
There are a few functional and morphological brain studies that have investigated the hypothesis that central mechanisms may be involved in dyspareunia. In vulvar vestibulitis patients with genital pain, A brain activation was identified on functional brain magnetic resonance imaging, which was similar to conditions with chronic pain Conditions such as fibromyalgia, chronic low back pain and irritable bowel syndrome ${ }^{[21]}$. The density of grey matter increased in the brain areas associated with pain modulation and stress In patients with vulvodynia using brain imaging techniques ${ }^{[22]}$.

\section{CONFLICT OF INTEREST}

There are no conflicts of interest.

\section{REFERENCES}

1. Jones GT, Atzeni F, Beasley M, Flüß E, Sarzi-Puttini P, Macfarlane GJ. The Prevalence of Fibromyalgia in the General Population: A Comparison of the American College of Rheumatology 1990, 2010, and Modified 2010 Classification Criteria. 2015;67(2):568-75.

2. Heidari F, Afshari M, Moosazadeh M. Prevalence of fibromyalgia in general population and patients, a systematic review and meta-analysis. Rheumatology international. 2017;37(9):1527-39.

3. Tuzun EH, Albayrak G, Eker L, Sozay S, Daskapan A. A comparison study of quality of life in women with fibromyalgia and myofascial pain syndrome. Disability and rehabilitation. 2004;26(4):198-202.

4. Kayhan F, Kucuk A, Satan Y, Ilgun E, Arslan S, Ilik F. Sexual dysfunction, mood, anxiety, and personality disorders in female patients with fibromyalgia. Neuropsychiatr Dis Treat. 2016;12:349-55.

5. Lewis RW, Fugl-Meyer KS, Corona G, Hayes RD, Laumann EO, Moreira ED, Jr., et al. Definitions/ epidemiology/risk factors for sexual dysfunction. The journal of sexual medicine. 2010;7(4 Pt 2):1598-607.

6. De Graaff AA, Van Lankveld J, Smits LJ, Van Beek JJ, Dunselman GAJ. Dyspareunia and depressive symptoms are associated with impaired sexual functioning in women with endometriosis, whereas sexual functioning in their male partners is not affected. Human Reproduction. 2016;31(11):2577-86.

7. Mitchell KR, Geary R, Graham CA, Datta J, Wellings $\mathrm{K}$, Sonnenberg P, et al. Painful sex (dyspareunia) in women: prevalence and associated factors in a British population probability survey. BJOG : an international journal of obstetrics and gynaecology. 2017;124(11):1689-97.

8. Sadownik LA. Etiology, diagnosis, and clinical management of vulvodynia. International journal of women's health. 2014;6:437-49.

9. Hampson JP, Reed BD, Clauw DJ, Bhavsar R,
Gracely RH, Haefner HK, et al. Augmented central pain processing in vulvodynia. The journal of pain: official journal of the American Pain Society. 2013;14(6):579-89.

10. Wolfe F, Clauw DJ, Fitzcharles MA, Goldenberg DL, Hauser W, Katz RS, et al. Fibromyalgia criteria and severity scales for clinical and epidemiological studies: a modification of the ACR Preliminary Diagnostic Criteria for Fibromyalgia. The Journal of rheumatology. 2011;38(6):1113-22.

11. Marinoff SC, Turner ML. Vulvar vestibulitis syndrome. Dermatologic clinics. 1992;10(2):435-44.

12. Hawker GA, Mian S, Kendzerska T, French M. Measures of adult pain: Visual Analog Scale for Pain (VAS Pain), Numeric Rating Scale for Pain (NRS Pain), McGill Pain Questionnaire (MPQ), Short-Form McGill Pain Questionnaire (SF-MPQ), Chronic Pain Grade Scale (CPGS), Short Form36 Bodily Pain Scale (SF-36 BPS), and Measure of Intermittent and Constant Osteoarthritis Pain (ICOAP). Arthritis Care Res (Hoboken). 2011;63 Suppl 11:S240-52.

13. Aalto AM, Elovainio M, Kivimaki M, Uutela A, Pirkola S. The Beck Depression Inventory and General Health Questionnaire as measures of depression in the general population: a validation study using the Composite International Diagnostic Interview as the gold standard. Psychiatry Res. 2012;197(1-2):163-71.

14. Maurer AJ, Lissounov A, Knezevic I, Candido KD, Knezevic NN. Pain and sex hormones: a review of current understanding. 2016;6(3):285-96.

15. Zolnoun D, Hartmann K, Lamvu G, As-Sanie S, Maixner W, Steege J. A conceptual model for the pathophysiology of vulvar vestibulitis syndrome. Obstetrical \& gynecological survey. 2006;61(6):395-401; quiz 23.

16. Giesecke J, Reed BD, Haefner HK, Giesecke T, Clauw DJ, Gracely RH. Quantitative sensory testing in vulvodynia patients and increased peripheral pressure pain sensitivity. Obstet Gynecol. 2004;104(1):126-33

17. Steege JF, Zolnoun DA. Evaluation and treatment of dyspareunia. Obstet Gynecol. 2009;113(5):1124-36.

18. Granot M, Friedman M, Yarnitsky D, Zimmer EZ. Enhancement of the perception of systemic pain in women with vulvar vestibulitis. Bjog. 2002;109(8):863-6.

19. Pukall CF, Baron M, Amsel R, Khalife S, Binik YM. Tender point examination in women with vulvar vestibulitis syndrome. The Clinical journal of pain. 2006;22(7):601-9.

20. Yap AU, Chua EK, Dworkin SF, Tan HH, 
Tan KB. Multiple pains and psychosocial functioning/psychologic distress in TMD patients. The International journal of prosthodontics. 2002;15(5):461-6.

21. Pukall CF, Strigo IA, Binik YM, Amsel R, Khalife $\mathrm{S}$, Bushnell MC. Neural correlates of painful genital touch in women with vulvar vestibulitis syndrome. Pain. 2005;115(1-2):118-27.

22. Schweinhardt P, Kuchinad A, Pukall CF, Bushnell MC. Increased gray matter density in young women with chronic vulvar pain. Pain. 2008;140(3):411-9. 